\title{
Krüppel-like factor expression and correlation with FAK, MMP-9 and E-cadherin expression in hepatocellular carcinoma
}

\author{
SHAOSHAN HAN ${ }^{1 *}$, LEI HAN ${ }^{2 *}$, HAO SUN $^{1}$, XIANFENG ZAN ${ }^{1}$, \\ ZHENYU ZHOU $^{1}$, KEDONG XU ${ }^{1}$, YINGMIN YAO ${ }^{1}$ and QINGGUANG LIU ${ }^{1}$ \\ ${ }^{1}$ Department of Hepatobiliary Surgery, The First Affiliated Hospital of College of Medicine; \\ ${ }^{2}$ Department of Immunology and Pathogenic Biology, College of Medicine, \\ Xi'an Jiaotong University, Xi'an, Shaanxi 710061, P.R. China
}

Received January 22, 2013; Accepted May 1, 2013

DOI: $10.3892 / \mathrm{mmr} .2013 .1471$

\begin{abstract}
Whether a focal adhesion kinase (FAK)-Krüppel-like factor 8 (KLF8)-matrix metalloproteinase (MMP)-9/E-cadherin signaling axis exists in hepatocellular carcinoma (HCC) remains unknown. In the present study, KLF8 expression and its clinicopathological significance in $\mathrm{HCC}$ was investigated to determine the correlation between KLF8 and FAK, MMP-9 and E-cadherin expression. Tissues were obtained from 60 surgically resected HCC and normal tumor-adjacent tissues. KLF8, FAK, MMP-9 and E-cadherin expression levels were examined by quantitative real-time reverse transcription polymerase chain reaction (qRT-PCR) and immunohistochemistry. In addition, KLF8, FAK, MMP-9 and E-cadherin protein expression levels were examined by western blot analysis in 5 portal vein cancer emboli and corresponding HCC tissues. The clinicopathological data of the HCC patients were retrospectively analyzed. KLF8 mRNA expression was found to be significantly upregulated in HCC tumor tissues compared with normal tumor-adjacent tissues $(\mathrm{P}<0.05)$. KLF8 protein was highly expressed in portal vein cancer emboli. KLF8 expression level was significantly higher in tumors with advanced TNM stages and vascular invasion compared with that in tumors with early TNM stage and absence of vascular invasion $(\mathrm{P}<0.05)$. KLF8 protein and mRNA expression in HCC positively correlated with that of FAK $(r=0.362, \mathrm{P}<0.001$; and $\mathrm{r}=0.377, \mathrm{P}<0.01$, respectively) and MMP-9 $(\mathrm{r}=0.392, \mathrm{P}<0.01$;
\end{abstract}

Correspondence to: Professor Qingguang Liu, Department of Hepatobiliary Surgery, The First Affiliated Hospital of College of Medicine, Xi'an Jiaotong University, 277 Yanta West Road, Xi'an, Shaanxi 710061, P.R. China

E-mail: liuqingguang@vip.sina.com

\section{*Contributed equally}

Key words: E-cadherin, focal adhesion kinase, hepatocellular carcinoma, Krüppel-like factor 8, matrix metalloproteinase-9 and $\mathrm{r}=0.336, \mathrm{P}<0.01$, respectively), but negatively correlated with E-cadherin $(r=-0.364, \mathrm{P}<0.01$; and $\mathrm{r}=-0.410, \mathrm{P}<0.01$, respectively). Results of the current study highlight a novel FAK-KLF8-MMP-9/E-cadherin signaling axis during HCC progression.

\section{Introduction}

Hepatocellular carcinoma (HCC) is a primary malignancy of the liver. It is the fifth most common type of cancer worldwide and a leading cause of cancer mortality, particularly in countries with a high prevalence of chronic hepatitis B virus (HBV) and HCV (1). Due to a poor therapeutic response to chemotherapy and radiotherapy, liver resection and transplantation are currently the main curative therapies for HCC. However, the long-term prognosis of the majority of patients with HCC remains extremely poor due to early recurrence and metastasis $(2,3)$. The majority of recurrences are associated with tumor cell invasion and intrahepatic metastasis (4). Understanding the molecular mechanisms associated with HCC invasion and metastasis and identifying potential biological markers of these processes are critical for the development of new therapeutic strategies.

Focal adhesion kinase (FAK), a cytoplasmic non-receptor tyrosine kinase, plays a central role in a number of cellular events, including cell proliferation, survival, migration and invasion (5). FAK is overexpressed and activated in a variety of human tumors, including HCC (6-9). Elevated FAK expression levels and activity are frequently associated with tumor metastasis and poor HCC patient prognosis (10). Krüppel-like factor 8 (KLF8) is a GT-box (CACCC)-binding dual-transcription factor that contains the $\mathrm{C} 2 \mathrm{H} 2$ zinc-finger motif and is critical for the regulation of cell cycle progression, cell differentiation and oncogenic transformation (11-13). Previous studies have demonstrated that KLF8 is markedly overexpressed in several types of human cancer, including breast cancer (11), renal carcinoma (12), gastric cancer (14) and HCC (15). Zhao et al (16) previously identified that KLF8 is a downstream target of FAK and the expression levels of KLF8 are specifically regulated by FAK-Src-phosphatidylinositol 3 kinase (PI3K) signaling in human ovarian cancer cells (17). 
KLF8 directly binds and represses the E-cadherin promoter, markedly inducing epithelial-to-mesenchymal transition (EMT), and enhances motility and invasiveness in breast cancer cells. Blocking KLF8 expression by RNA interference restores E-cadherin expression in the cancer cells and markedly inhibits cell invasion (11). KLF8 overexpression has also been found to induce an increase in MMP-9 expression and activity. KLF8 directly binds and activates the human MMP-9 gene promoter (18). These observations indicate that the FAK-KLF8-MMP-9/E-cadherin signaling axis is important for cancer progression, enabling tumor cells to gain EMT phenotypes and increase MMP-9 levels, leading to increased invasion and metastasis.

KLF8 has also been reported to play an oncogenic role in HCC. Li et al (15) found that KLF8 promotes HCC cell proliferation and invasion, inhibits apoptosis and induces EMT. However, the molecular mechanisms by which KLF8 enhances HCC cell invasion and metastasis remain poorly understood. We previously hypothesized that the FAK-KLF8-MMP-9/E-cadherin signaling axis plays a vital role in HCC progression. In the present study, KLF8 expression and its clinicopathological significance in HCC was investigated using quantitative real-time reverse transcription polymerase chain reaction (qRT-PCR) and immunohistochemistry. In addition, the correlation between KLF8 and FAK, MMP-9 and E-cadherin expression was determined in HCC.

\section{Materials and methods}

Patients and specimens. Sixty fresh tumor samples for qRT-PCR and immunohistochemistry were randomly collected from HCC patients who underwent curative resection between 2008 and 2010 at the First Affiliated Hospital of Medical College, Xi'an Jiaotong University (Xi'an, China). For western blot analysis, 10 invasive tumor tissues were selected from the 60 cases. Resected tumors and corresponding non-tumor tissues specimens ( $>2 \mathrm{~cm}$ away from the tumor) were immediately cut from the resected liver and subdivided into 2 portions. One portion was rapidly frozen in liquid nitrogen and stored at $-80^{\circ} \mathrm{C}$ until use and the other was fixed in buffered paraformaldehyde for immunohistochemical staining. None of the patients had received radiotherapy or chemotherapy prior to sampling. Clinical data were obtained from medical records. Histopathological Edmonson classification, clinical tumor-node-metastasis (TNM) grading, maximum tumor diameter and normal tumor-adjacent tissues were all confirmed by an experienced pathologist who was blinded to the clinical information. Written informed consent was obtained from all patients. The Xi'an Jiaotong University Ethics Committee approved all protocols according to the Helsinki Declaration of 1975.

Immunohistochemical staining. Immunohistochemical staining was performed on paraformaldehyde-fixed paraffin sections (4 $\mu \mathrm{m})$, as described previously (19). In brief, sections were dewaxed and rehydrated, and antigens were retrieved in citrate buffer. Endogenous peroxidase activity was blocked for 10 min using $3.0 \%$ hydrogen peroxide. Goat serum (10\%) was applied to the sections to prevent non-specific binding. The sections were then separately incubated with the following primary antibodies at $4^{\circ} \mathrm{C}$ overnight: rabbit anti-FAK (1:200; Cell Signaling Technology, Inc., Danvers, MA, USA), rabbit anti-KLF8 (1:200; Aviva Systems Biology, San Diego, CA, USA), mouse anti-E-cadherin (1:100) and rabbit anti-MMP-9 (1:200; both Santa Cruz Biotechnology, Santa Cruz, CA, USA). The primary antibody was detected using biotinylated secondary antibodies (Beijing Zhongshan Golden Bridge Biotechnology Co., Ltd., Beijing, China) according to the manufacturer's instructions. Sections were visualized with diaminobenzidine, counterstained with hematoxylin, dehydrated in alcohol and xylene and mounted onto glass slides. For negative controls, primary antibody was replaced with PBS.

All sections were assessed independently by two experienced pathologists. The staining results for the four proteins (FAK, KLF8, E-cadherin and MMP-9) were semi-quantitatively expressed by an immunohistochemical score combined with the percentage of liver cells revealing specific immunoreactivity. Staining intensity was expressed by four grades as follows: 0 , none; 1 , weak; 2 , moderate; and 3 , strong. The percentage of positive liver cells was expressed by the following grades: $0,<10 \% ; 1,10-25 \% ; 2,26-50 \% ; 3,51-75 \%$; and $4,>75 \%$. The staining intensity and average percentage of positive liver cells were assayed for 5 independent high magnification fields. The total score was calculated by multiplying the staining intensity and the percentage score of positive liver cells. Specimen staining was assessed as total score: negative $(-)$, score 0 ; weakly positive $(+)$, score $1-4$; positive $(++)$, score 5-8; strongly positive $(+++)$, score 9-12.

Western blot analysis. Proteins were extracted from the portal vein cancer emboli and corresponding HCC tissues of 5 patients and lysed in RIPA buffer containing protease inhibitor cocktail and PMSF on ice. Protein concentration was quantified using a BCA Protein Assay kit (Pierce Biotechnology, Inc., Rockford, IL, USA) according to the manufacturer's instructions. Proteins suspended in loading buffer were heated at $95^{\circ} \mathrm{C}$ for $5 \mathrm{~min}$ for denaturation. Equal amounts of proteins ( $20 \mu \mathrm{g} / \mathrm{lane})$ were separated by sodium dodecyl sulfate-polyacrylamide gel electrophoresis and transferred to polyvinylidene fluoride membranes (Millipore, Billerica, MA, USA). Following blocking for $1 \mathrm{~h}$ with $5 \%$ non-fat milk and TBST washes, the membranes were incubated overnight at $4{ }^{\circ} \mathrm{C}$ with primary antibodies against FAK (1:1,000), KLF8 (1:500), E-cadherin (1:1,000), MMP-9 $(1: 1,000)$ and $\beta$-actin $(1: 2,000$; Santa Cruz Biotechnology). Subsequently, the membranes were incubated with goat anti-mouse or anti-rabbit horseradish peroxidase-conjugated secondary antibodies (1:10,000; Santa Cruz Biotechnology) for $1 \mathrm{~h}$ at room temperature. Blots were then visualized using the Super Signal West Pico chemiluminescent substrate kit (Millipore) and exposed to X-ray films.

$q R T-P C R$. Total RNA was isolated from the frozen specimens stored in liquid nitrogen using the RNAfast 200 purification kit (Fastagen Biotech, Shanghai, China). $\mathrm{A}_{260 / 280}$ optical density values of the RNA samples were between 1.8 and 2.1. RNA integrity was confirmed by the presence of intact $18 \mathrm{~S}$ and $28 \mathrm{~S}$ bands on $2 \%$ agarose gels. Next, total RNA was reverse 
Table I. Primer sequences for qRT-PCR.

\begin{tabular}{lllc}
\hline Gene & \multicolumn{1}{c}{ Forward (5'-3') } & \multicolumn{1}{c}{ Reverse (5'-3') } & Products (bp) \\
\hline FAK & ACATTATTGGCCACTGTGGATGAG & GGCCAGTTTCATCTTGTTGATGAG & 126 \\
KLF8 & CTGGACAGTTGCTTATGGCATC & CTGCCTAATCTACCCAATCAGTTCA & 169 \\
E-cadherin & AGTGGGCACAGATGGTGTGA & TAGGTGGAGTCCCAGGCGTA & 93 \\
MMP-9 & CCTGGGCAGATTCCAAACCT & GCAAGTCTTCCGAGTAGTTTGGAT & 89 \\
$\beta$-actin & TGGCACCCAGCACAATGAA & CTAAGTCATAGTCCGCCTAGAAGCA & 186 \\
\hline
\end{tabular}

FAK, focal adhesion kinase; KLF8, Krüppel-like factor 8; MMP-9, matrix metalloproeteinase 9; qRT-PCR, quantitative real-time reverse transcription polymerase chain reaction.

transcribed at $37^{\circ} \mathrm{C}$ for $15 \mathrm{~min}$ and incubated at $85^{\circ} \mathrm{C}$ for 5 sec using PrimeScript RT reagent (Takara Biotechnology, Shiga, Japan). cDNA was used for qRT-PCR analysis with SYBR-Green PCR master mix (Takara Biotechnology) and the BD IQ5 cycler (BD Biosciences, Franklin Lakes, NJ, USA) according to the manufacturer's instructions. The primer sequences used for the detection of FAK, KLF8, MMP-9, E-cadherin and $\beta$-actin are summarized in Table I. PCR was performed under the following conditions: 1 cycle at $95^{\circ} \mathrm{C}$ for $5 \mathrm{~min}$, then 40 cycles at $95^{\circ} \mathrm{C}$ for $30 \mathrm{sec}$ and $60^{\circ} \mathrm{C}$ for $30 \mathrm{sec}$. $\beta$-actin was used as an internal control. Relative mRNA levels were calculated based on the threshold cycle (CT) values, which were corrected based on $\beta$-actin expression, according to the equation: $2^{-\Delta \mathrm{CT}}\left(\Delta \mathrm{CT}=\mathrm{CT}_{\text {target gene }}-\mathrm{Ct}_{\beta \text {-actin }}\right)$.

Statistical analysis. Statistical analyses were performed with the SPSS 16.0 software (SPSS, Inc., Chicago, IL, USA). For continuous variables, data are expressed as the mean \pm SD. KLF8 mRNA expression levels in the tumor and adjacent non-tumorous tissues were compared by the paired t-test. Clinicopathological parameters and KLF8 mRNA expression correlations were analyzed using the Mann-Whitney U test. Clinicopathological parameters and KLF8 protein expression correlations were analyzed using the Spearman's rank correlation coefficient test. The correlation between KLF8 mRNA expression and FAK, MMP-9 and E-cadherin mRNA expression was analyzed using the Pearson correlation coefficient test and the correlation between KLF8 protein expression and FAK, MMP-9 and E-cadherin protein expression was analyzed using Spearman's rank correlation coefficient test. $\mathrm{P}<0.05$ was considered to indicate a statistically significant difference.

\section{Results}

KLF8 mRNA and protein expression in HCC and adjacent non-tumorous tissues. KLF8 mRNA expression in 60 pairs of HCC tumor and adjacent non-tumorous tissues was detected by qRT-PCR. The expression of KLF8 mRNA in HCC tissues was markedly upregulated compared with adjacent non-tumorous tissues $(\mathrm{P}<0.05$; Fig. 1A). Immunohistochemical staining was performed in the same tissues to detect KLF8 protein expression. Specifically, $51.6 \%(n=31)$ of the HCC tumor tissues and $41.6 \%(n=25)$ of the adjacent non-tumorous tissues revealed KLF8-positive staining. The majority of positive cells in HCC tumor tissues

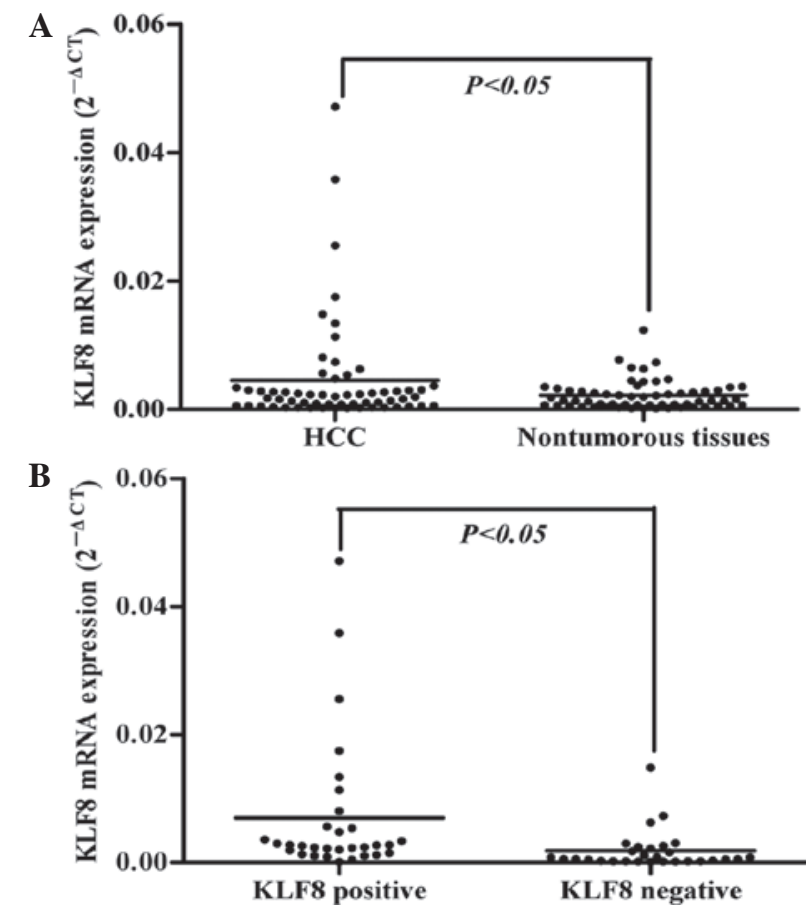

Figure 1. KLF8 mRNA expression in HCC tissue samples. (A) KLF8 mRNA expression in 60 pairs of HCC tumor and adjacent non-tumorous tissues, as determined by qRT-PCR analysis. (B) KLF8 mRNA expression in tumor tissues with positive $(n=31)$ and negative $(n=29)$ staining, as determined by immunohistochemistry. KLF8, Krüppel-like factor 8; HCC, hepatocellular carcinoma; qRT-PCR, quantitative real-time reverse transcription polymerase chain reaction.

revealed diffuse cytoplasmic staining and intense nuclear staining of KLF8, while adjacent non-tumorous tissues mainly exhibited diffuse cytoplasmic staining (Fig. 2). These observations indicate that KLF8 nuclear translocation is required during $\mathrm{HCC}$ progression. We also investigated whether the higher expression of KLF8 in HCC tissues was consistent at the mRNA and protein levels. As demonstrated in Fig. 1B, by comparing the mRNA levels of KLF8 in tumor tissues with positive staining $(n=31)$ to those with negative $(n=29)$ staining, as determined by immunohistochemistry, samples with positive protein staining were found to have significantly higher mRNA expression than the negative group $(\mathrm{P}<0.05)$, indicative of a consistency between KLF8 mRNA and protein levels during HCC development. 
Table II. Correlation between KLF8 expression and clinicopathological characteristics in 60 HCC patients.

\begin{tabular}{|c|c|c|c|c|c|c|c|c|c|}
\hline \multirow{2}{*}{$\begin{array}{l}\text { Clinicopathological } \\
\text { parameters }\end{array}$} & \multirow[b]{2}{*}{$\mathrm{n}$} & \multirow{2}{*}{$\begin{array}{l}\text { KLF8 mRNA } \\
(\text { mean } \pm \text { SD) }\end{array}$} & \multirow[b]{2}{*}{ P-value } & \multicolumn{4}{|c|}{ KLF8 protein expression } & \multirow[b]{2}{*}{ r-value } & \multirow[b]{2}{*}{ P-value } \\
\hline & & & & - & + & ++ & +++ & & \\
\hline \multicolumn{10}{|l|}{ Age, years } \\
\hline$\leq 55$ & 27 & $0.0053 \pm 0.0084$ & 0.181 & 11 & 7 & 7 & 2 & -0.119 & 0.367 \\
\hline$>55$ & 33 & $0.0039 \pm 0.0084$ & & 18 & 6 & 7 & 2 & & \\
\hline \multicolumn{10}{|l|}{ Gender } \\
\hline Male & 49 & $0.0043 \pm 0.0080$ & 0.731 & 23 & 11 & 12 & 3 & 0.043 & 0.745 \\
\hline Female & 11 & $0.0056 \pm 0.0105$ & & 6 & 2 & 2 & 1 & & \\
\hline \multicolumn{10}{|l|}{ Tumor size, $\mathrm{cm}$} \\
\hline$<5$ & 18 & $0.0044 \pm 0.0069$ & 0.729 & 8 & 4 & 3 & 3 & -0.088 & 0.503 \\
\hline$\geq 5$ & 42 & $0.0046 \pm 0.0090$ & & 21 & 9 & 11 & 1 & & \\
\hline \multicolumn{10}{|l|}{ Tumor number } \\
\hline Solitary & 53 & $0.0049 \pm 0.0089$ & 0.276 & 26 & 12 & 12 & 3 & 0.081 & 0.540 \\
\hline Multiple & 7 & $0.0014 \pm 0.0012$ & & 3 & 1 & 2 & 1 & & \\
\hline \multicolumn{10}{|l|}{ Edmonson's staging } \\
\hline I-II & 45 & $0.0023 \pm 0.0029$ & 0.069 & 22 & 11 & 10 & 2 & 0.082 & 0.531 \\
\hline III-IV & 15 & $0.0104 \pm 0.0143$ & & 7 & 2 & 4 & 2 & & \\
\hline \multicolumn{10}{|l|}{ TNM staging } \\
\hline I & 40 & $0.0029 \pm 0.0045$ & 0.198 & 23 & 7 & 9 & 1 & 0.261 & 0.044 \\
\hline II-III & 20 & $0.0078 \pm 0.0127$ & & 6 & 6 & 5 & 3 & & \\
\hline \multicolumn{10}{|l|}{ Vascular invasion } \\
\hline Absent & 47 & $0.0027 \pm 0.0042$ & 0.047 & 27 & 8 & 11 & 1 & 0.346 & 0.007 \\
\hline Present & 13 & $0.0111 \pm 0.0148$ & & 2 & 5 & 3 & 3 & & \\
\hline \multicolumn{10}{|l|}{ AFP, $\mathrm{ng} / \mathrm{ml}$} \\
\hline$\leq 20$ & 23 & $0.0045 \pm 0.0086$ & 0.637 & 11 & 4 & 7 & 1 & -0.027 & 0.84 \\
\hline$>20$ & 37 & $0.0045 \pm 0.0084$ & & 18 & 9 & 7 & 3 & & \\
\hline \multicolumn{10}{|l|}{ HBsAg } \\
\hline Positive & 44 & $0.0045 \pm 0.0086$ & 0.688 & 21 & 12 & 9 & 2 & -0.076 & 0.563 \\
\hline Negative & 16 & $0.0041 \pm 0.0071$ & & 8 & 1 & 5 & 2 & & \\
\hline
\end{tabular}

AFP, fetoprotein; TNM, tumor-node-metastasis; HBsAg, hepatitis B surface antigen; KLF8, Krüppel-like factor 8; HCC, hepatocellular carcinoma.
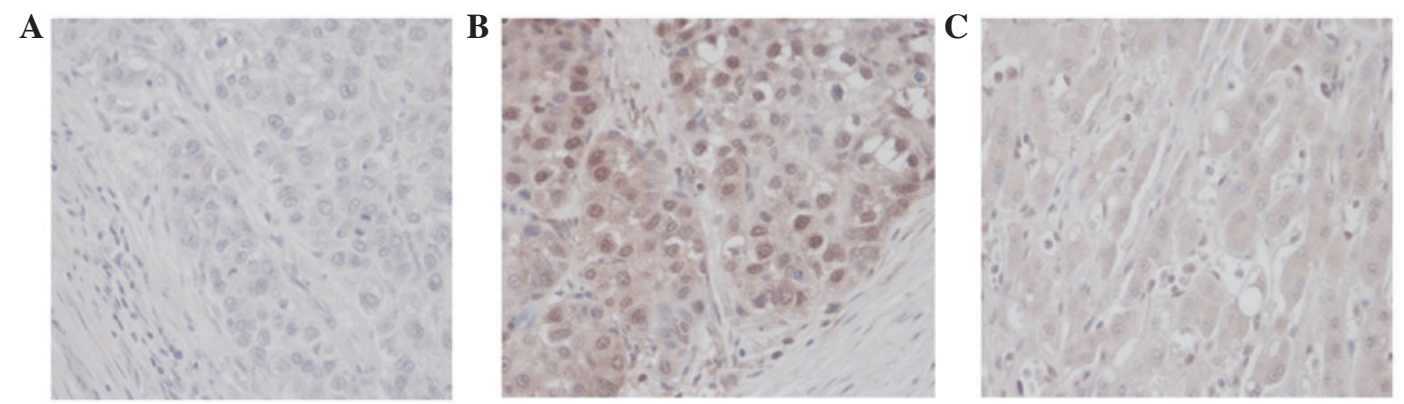

Figure 2. Immunohistochemical staining of KLF8 in HCC tissues and adjacent non-tumorous tissues. (A) A negative control was used to demonstrate the specificity of the antibody. (B) Positive staining of KLF8 in HCC tissues was localized mainly in the nucleus. (C) Positive staining of KLF8 in adjacent non-tumorous tissues revealed diffuse cytoplasmic distribution. KLF8, Krüppel-like factor 8; HCC, hepatocellular carcinoma.

Correlation between KLF8 expression in HCC tissues and clinicopathological characteristics. To assess the clinical significance of KLF8 expression in HCC, the correlation between KLF8 expression and the clinicopathological characteristics of the $60 \mathrm{HCC}$ patients was statistically analyzed, and the results are presented in Table II. HCC tissues with 
Table III. Correlation between KLF8 protein expression and FAK, E-cadherin and MMP-9 protein expression in $60 \mathrm{HCC}$ patients

\begin{tabular}{|c|c|c|c|c|c|c|c|c|c|c|c|c|c|c|c|c|c|c|}
\hline \multirow[b]{2}{*}{ KLF8 } & \multicolumn{4}{|c|}{ FAK } & \multirow[b]{2}{*}{ r-value } & \multirow[b]{2}{*}{$\mathrm{P}$-value } & \multicolumn{4}{|c|}{ MMP-9 } & \multirow[b]{2}{*}{ r-value } & \multirow[b]{2}{*}{ P-value } & \multicolumn{4}{|c|}{ E-cadherin } & \multirow[b]{2}{*}{ r-value } & \multirow[b]{2}{*}{ P-value } \\
\hline & - & + & ++ & +++ & & & - & + & ++ & +++ & & & - & + & ++ & +++ & & \\
\hline 0 & 18 & 7 & 3 & 1 & 0.377 & 0.003 & 9 & 9 & 9 & 2 & 0.336 & 0.009 & 11 & 7 & 7 & 4 & -0.410 & 0.001 \\
\hline+ & 6 & 7 & 0 & 0 & & & 5 & 4 & 4 & 0 & & & 6 & 3 & 2 & 2 & & \\
\hline++ & 4 & 4 & 5 & 1 & & & 2 & 3 & 3 & 6 & & & 12 & 1 & 1 & 0 & & \\
\hline+++ & 0 & 2 & 1 & 1 & & & 0 & 0 & 2 & 2 & & & 4 & 0 & 0 & 0 & & \\
\hline
\end{tabular}

FAK, focal adhesion kinase; KLF8, Krüppel-like factor 8; MMP-9, matrix metalloproeteinase-9; HCC, hepatocellular carcinoma.

vascular invasion exhibited higher KLF8 mRNA and protein expression levels than those without vascular invasion $(\mathrm{P}<0.05)$. HCC tissues with advanced TNM staging exhibited higher KLF8 protein expression levels than those with a lower TNM staging $(r=0.261, \mathrm{P}<0.05)$. However, no significant correlation was observed between KLF8 expression and age, gender, HBV infection, fetoprotein (AFP), tumor number and tumor size. In addition, western blot analysis revealed that KLF8 protein levels were higher in portal vein cancer emboli compared with their corresponding primary $\mathrm{HCC}$ tissues (Fig. 3).

KLF8 expression in HCC tissues positively correlates with FAK expression. KLF8 and FAK mRNA expression levels were determined by qRT-PCR in 60 HCC tissues. KLF8 mRNA expression was found to positively correlate with FAK mRNA expression levels in the HCC tissues $(\mathrm{r}=0.362$, $\mathrm{P}<0.001)$, as assessed by Pearson correlation coefficient test (Fig. 4A). KLF8 and FAK protein expression was determined by immunohistochemical staining. FAK protein was positively stained in the cytoplasm of carcinoma cells and $53.3 \%(n=32)$ of the HCC tumor samples revealed positive staining of FAK protein. KLF8 protein expression levels positively correlated with FAK protein expression levels in the HCC tissues ( $\mathrm{r}=0.377, \mathrm{P}<0.01)$, as assessed by Spearman's rank correlation coefficient test (Table III, Fig. 5).

KLF8 expression in HCC tissues positively correlates with MMP-9 expression. qRT-PCR demonstrated that KLF8 mRNA expression levels positively correlated with MMP-9 mRNA expression levels in the HCC tissues ( $\mathrm{r}=0.392, \mathrm{P}<0.01)$, as assessed by Pearson correlation coefficient test (Fig. 4B). Immunohistochemical staining revealed that MMP-9 protein was diffuse with moderate or strong staining in the cytoplasm of tumor cells. Positive staining of MMP-9 protein was found in $73.3 \%(n=44)$ of the HCC tissues. Consistent with mRNA expression levels, KLF8 protein expression positively correlated with MMP-9 protein expression in the HCC tissues $(r=0.336, P<0.01$; Table III, Fig. 5).

KLF8 expression in HCC tissues negatively correlates with E-cadherin expression. KLF8 mRNA expression levels were found to negatively correlate with E-cadherin mRNA expression levels in the HCC tissues $(\mathrm{r}=-0.364, \mathrm{P}<0.01)$, as assessed by Pearson correlation coefficient test (Fig. 2C).

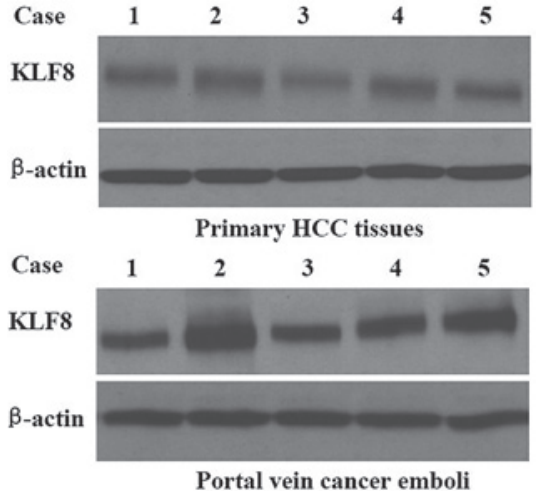

Figure 3. Western blot analysis of KLF8 protein expression in portal vein cancer emboli and their corresponding HCC tissues. Higher expression levels of KLF8 were detected in portal vein cancer emboli compared with corresponding primary HCC tissues. Cases were numbered as 1, 2, 3, 4 and 5. $\beta$-actin was used as internal control. KLF8, Krüppel-like factor 8; HCC, hepatocellular carcinoma.

Immunohistochemical analysis revealed that positive E-cadherin protein staining was largely presented in the membranes of carcinoma cells. The positive staining of E-cadherin protein was found in $45 \%(n=27)$ of the HCC tissues. KLF8 protein expression levels negatively correlated with E-cadherin protein expression levels in the HCC tissues $(r=-0.410, P<0.01$; Table III, Fig. 5).

\section{Discussion}

The long-term prognosis of the majority of patients with $\mathrm{HCC}$ remains extremely poor due to early recurrences and metastases. HCC metastasis requires invasive cells to detach from the localized tumors by EMT and degrade the extracellular matrix using proteases, including MMPs. Following this, these cells must survive the circulation as circulating tumor cells and colonize distant locations $(20,21)$. Understanding the molecular mechanisms underlying each of these steps is crucial for targeting metastatic cells at early stages to improve patient survival.

KLF8 has recently emerged as an important oncogenic transcription factor and is overexpressed in several types of human cancer (11-15). In the present study, KLF8 mRNA expression was found to be significantly upregulated in HCC tumor tissues compared with tumor-adjacent tissues. KLF8 

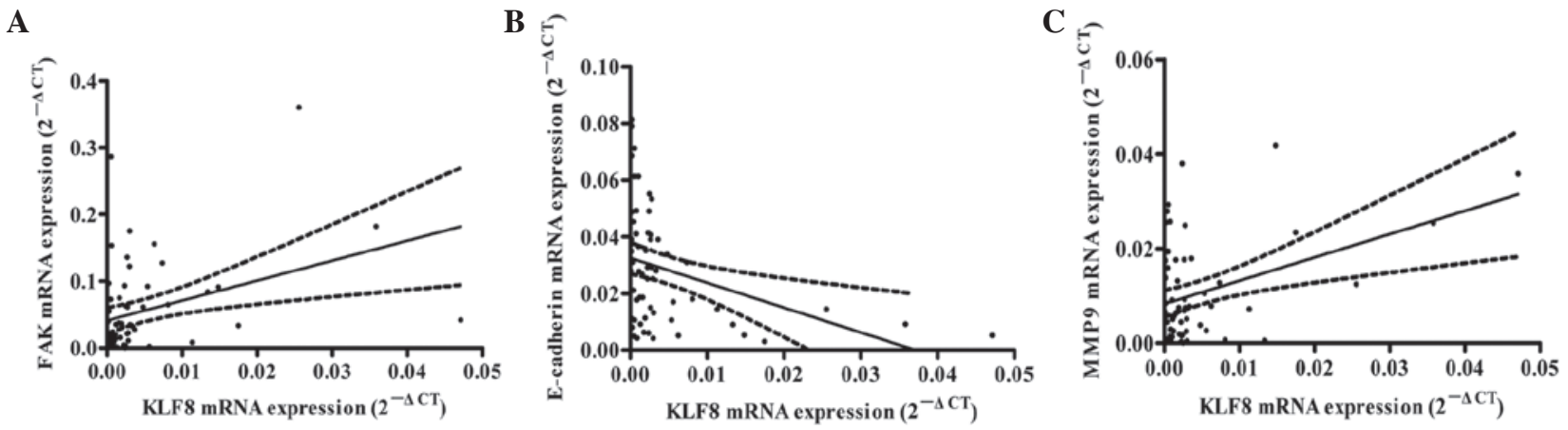

Figure 4. Correlation between KLF8 and FAK, E-cadherin and MMP-9 mRNA expression in 60 HCC patients. KLF8 mRNA expression was positively correlated with (A) FAK ( $\mathrm{r}=0.362, \mathrm{P}<0.001)$ and (B) MMP-9 mRNA expression $(\mathrm{r}=0.392, \mathrm{P}<0.01)$ and $(\mathrm{C})$ negatively correlated with E-cadherin mRNA expression $(\mathrm{r}=-0.364, \mathrm{P}<0.01)$. FAK, focal adhesion kinase; KLF8, Krüppel-like factor 8; MMP-9, matrix metalloproeteinase 9; HCC, hepatocellular carcinoma.
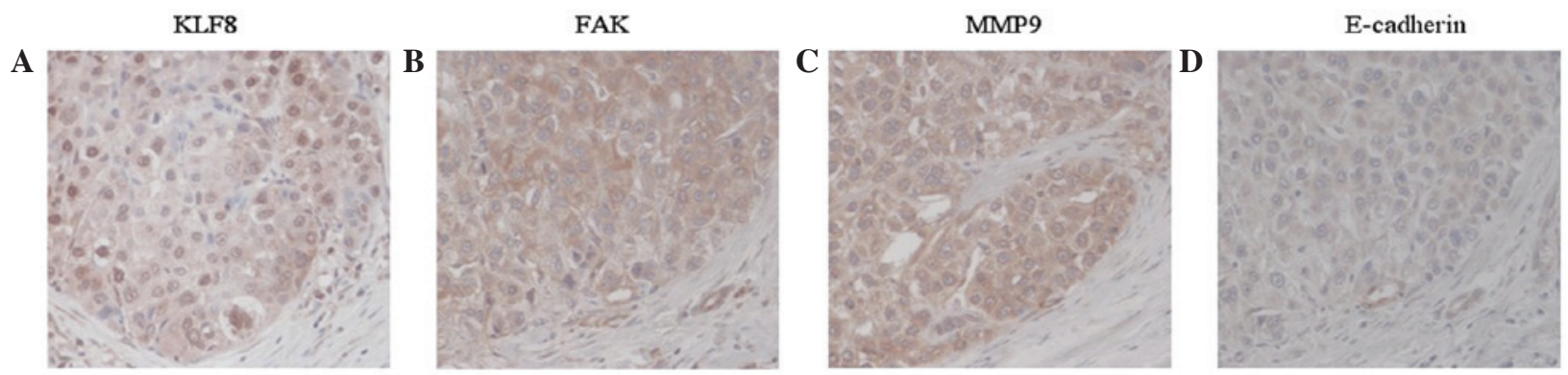

$\mathbf{E}$

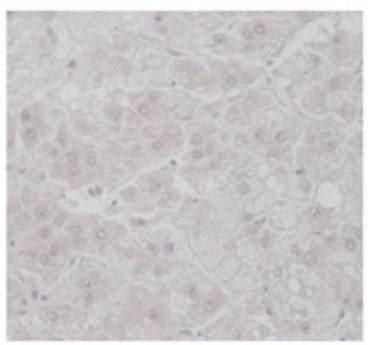

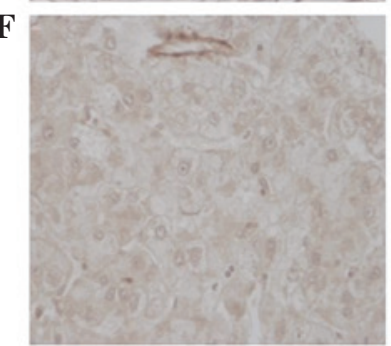
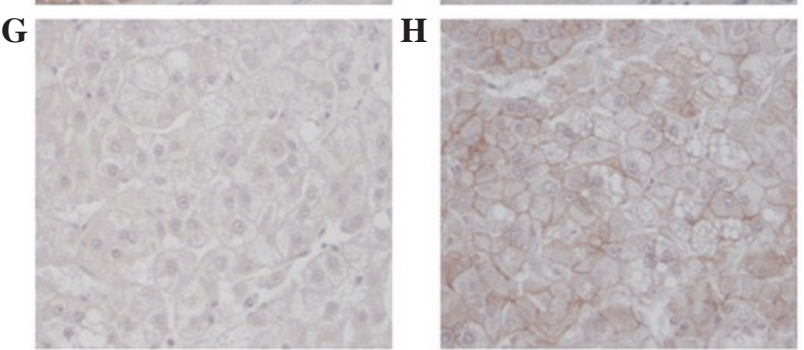

Figure 5. Immunohistochemical staining of KLF8, FAK, MMP-9 and E-cadherin in HCC. (A) High KLF8 protein expression was consistent with high (B) FAK and (C) MMP-9 protein expression and (D) no detectable E-cadherin. (E) Low KLF8 protein expression was consistent with (F) low FAK and (G) MMP-9 protein expression and (H) strong E-cadherin protein expression (magnification, x400). FAK, focal adhesion kinase; KLF8, Krüppel-like factor 8; MMP-9, matrix metalloproeteinase 9; HCC, hepatocellular carcinoma.

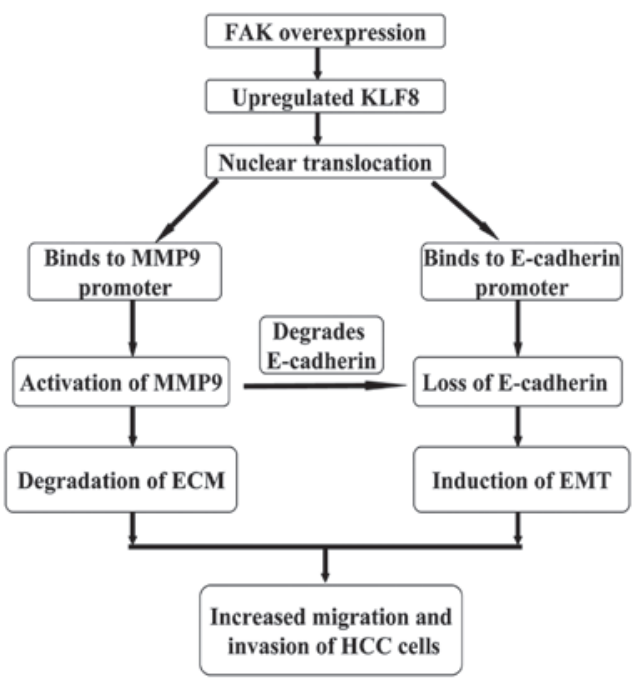

Figure 6. Schematic representation of HCC FAK-KLF8-MMP-9/E-cadherin signaling activation. FAK, focal adhesion kinase; KLF8, Krüppel-like factor 8; MMP-9, matrix metalloproeteinase 9; HCC, hepatocellular carcinoma; EMT, epithelial-mesenchymal transition; ECM, extracellular matrix. expression level was significantly higher in tumors with advanced TNM stages and vascular invasion compared with that in tumors with early TNM stage and absence of vascular invasion $(\mathrm{P}<0.05)$. In addition, higher expression levels of KLF8 protein were detected in portal vein cancer emboli compared with corresponding primary HCC tissues. These results indicate that increased KLF8 expression correlates with more invasive $\mathrm{HCC}$ phenotypes and a poorer prognosis.

Next, the correlation between KLF8 expression and its possible upstream and downstream factors was analyzed in 60 HCC tissues. FAK, a cytoplasmic non-receptor tyrosine kinase, plays a key role in the regulation of cell proliferation, survival, migration and invasion in HCC. High levels of FAK expression were found to correlate with early metastasis and poor prognosis in HCC patients who underwent curative surgery (22). KLF8 was previously identified as a downstream target of FAK and KLF8 mRNA levels are upregulated by FAK through the PI3K-Akt-Sp1 signaling pathway in ovarian cancer cells $(11,16)$. In the present study, KLF8 expression levels were found to positively correlate with FAK expression 
in HCC tissues. These observations indicated that KLF8 may also be a downstream target of FAK in HCC cells and is regulated by FAK.

MMP-9 is a member of the MMP family that degrades denatured collagens and type IV collagen present in the basement membrane. Thus, MMP-9 is critical for tumor cell invasion and metastasis (23). MMP-9 is significantly upregulated in several types of cancer, including $\mathrm{HCC}$, and is associated with tumor progression and poor survival in HCC patients $(24,25)$. KLF8 directly binds and activates the MMP-9 gene promoter, leading to a marked increase in MMP-9 expression and activity in human breast cancer cells (18). In the current study, KLF8 expression levels were found to positively correlate with MMP-9 expression in HCC tissues. The positive correlation between KLF8 and MMP-9 indicated that MMP-9 may represent a downstream target of KLF8 in HCC cells, and the increased activity of MMP-9 in HCC cells may be caused, in part, by upregulated KLF8 expression.

During EMT, non-invasive and non-metastatic tumor cells lose their epithelial phenotype, acquire invasive properties, infiltrate surrounding tissues and metastasize to secondary sites, and this process is considered to be a crucial step of metastasis (26). Studies have demonstrated that EMT frequently occurs in HCC and is involved in tumorigenesis and metastasis $(27,28)$. Loss of expression of the epithelial marker, E-cadherin, is the hallmark of EMT, resulting in reduced epithelial integrity and polarized function. The downregulated expression of E-cadherin is common in HCC and has been found to be associated with hypermethylation of the E-cadherin promoter (29), HBV infection (30) and the aberrant activity of several transcription factors, including Snail and Twist (31). Previously, KLF8 was found to directly bind and repress the promoter of E-cadherin, markedly inducing EMT and enhancing motility and invasiveness in breast cancer cells (11). In the present study, KLF8 expression levels were found to negatively correlate with E-cadherin expression in HCC tissues. KLF8 was highly expressed in portal vein cancer emboli, while E-cadherin expression was low (data not shown). The negative correlation between KLF8 and E-cadherin indicated that E-cadherin may be regulated by KLF8 in HCC cells and the loss of E-cadherin expression may be caused, in part, by KLF8 overexpression. Notably, MMP-9 degrades E-cadherin into soluble E-cadherin, thus suppressing E-cadherin activity and inducing EMT (32). These observations indicate that a loss of E-cadherin expression and activity may be a result of the KLF8-mediated repression of E-cadherin and induction of MMP-9.

Results of the present study demonstrate the importance of the FAK-KLF8-MMP-9/E-cadherin signaling axis during HCC progression (Fig. 6). Overexpression of FAK in HCC cells leads to the upregulation of KLF8 expression and increased KLF8 nuclear translocation. KLF8 binds to the promoters of MMP-9 and E-cadherin, inducing marked increases in MMP-9 activity and a loss of E-cadherin expression. In this manner, HCC cells gain the ability to degrade the extracellular matrix and undergo EMT.

\section{Acknowledgements}

The present study was supported by a grant from the National Natural Science Foundation of China (no. 81272645).

\section{References}

1. Farazi PA and DePinho RA: Hepatocellular carcinoma pathogenesis: from genes to environment. Nat Rev Cancer 6: 674-687, 2006.

2. Eguchi S, Kanematsu T, Arii S, et al: Recurrence-free survival more than 10 years after liver resection for hepatocellular carcinoma. Br J Surg 98: 552-557, 2011.

3. El-Serag HB, Marrero JA, Rudolph L and Reddy KR: Diagnosis and treatment of hepatocellular carcinoma. Gastroenterology 134 : 1752-1763, 2008

4. Tang ZY, Ye SL, Liu YK, et al: A decade's studies on metastasis of hepatocellular carcinoma. J Cancer Res Clin Oncol 130: 187-196, 2004.

5. McLean GW, Carragher NO, Avizienyte E, Evans J, Brunton VG and Frame MC: The role of focal-adhesion kinase in cancer. A new therapeutic opportunity. Nat Rev Cancer 5: 505-515, 2005.

6. Watermann DO, Gabriel B, Jager M, et al: Specific induction of pp125 focal adhesion kinase in human breast cancer. Br J Cancer 93: 694-698, 2005.

7. Miyazaki T, Kato H, Nakajima M, et al: FAK overexpression is correlated with tumour invasiveness and lymph node metastasis in oesophageal squamous cell carcinoma. Br J Cancer 89: 140-145, 2003.

8. Rovin JD, Frierson HF Jr, Ledinh W, Parsons JT and Adams RB: Expression of focal adhesion kinase in normal and pathologic human prostate tissues. Prostate 53: 124-132, 2002.

9. Aronsohn MS, Brown HM, Hauptman G and Kornberg LJ: Expression of focal adhesion kinase and phosphorylated focal adhesion kinase in squamous cell carcinoma of the larynx. Laryngoscope 113: 1944-1948, 2003.

10. Itoh S, Maeda T, Shimada M, et al: Role of expression of focal adhesion kinase in progression of hepatocellular carcinoma. Clin Cancer Res 10: 2812-2817, 2004

11. Wang XH,Zheng MZ, Liu G, et al: Krüppel-like factor 8 induces epithelial to mesenchymal transition and epithelial cell invasion. Cancer Res 67: 7184-7193, 2007.

12. Fu WJ, Li JC, Wu XY, et al: Small interference RNA targeting Krüppel-like factor 8 inhibits the renal carcinoma 786-0 cells growth in vitro and in vivo. J Cancer Res Clin Oncol 136: $1255-1265,2010$

13. Wei H, Wang X, Gan B, et al: Sumoylation delimits KLF8 transcriptional activity associated with the cell cycle regulation. J Biol Chem 281: 16664-16671, 2006.

14. Liu L, Liu N, Xu M, et al: Lentivirus-delivered Krüppel-like factor 8 small interfering RNA inhibits gastric cancer cell growth in vitro and in vivo. Tumour Biol 33: 53-61, 2012.

15. Li JC, Yang XR, Sun HX, et al: Up-regulation of Krüppel-like factor 8 promotes tumor invasion and indicates poor prognosis for hepatocellular carcinoma. Gastroenterology 139: 2146-2157, 2010.

16. Zhao J, Bian ZC, Yee K, Chen BP, Chien S and Guan JL: Identification of transcription factor KLF8 as a downstream target of focal adhesion kinase in its regulation of cyclin D1 and cell cycle progression. Mol Cell 11: 1503-1515, 2003.

17. Wang X, Urvalek AM, Liu J and Zhao J: Activation of KLF8 transcription by focal adhesion kinase in human ovarian epithelial and cancer cells. J Biol Chem 283: 13934-13942, 2008.

18. Wang X, Lu H, Urvalek AM, et al: KLF8 promotes human breast cancer cell invasion and metastasis by transcriptional activation of MMP-9. Oncogene 30: 1901-1911, 2011.

19. Tu K, Zheng X, Zan X, Han S, Yao Y and Liu Q: Evaluation of Fbxw7 expression and its correlation with the expression of c-Myc, cyclin E and p53 in human hepatocellular carcinoma. Hepatol Res 42: 904-910, 2012.

20. Pantel K and Brakenhoff RH: Dissecting the metastatic cascade. Nat Rev Cancer 4: 448-456, 2004.

21. Huber MA, Kraut N and Beug H: Molecular requirements for epithelial-mesenchymal transition during tumor progression. Curr Opin Cell Biol 17: 548-558, 2005.

22. Fujii T, Koshikawa K, Nomoto S, et al: Focal adhesion kinase is overexpressed in hepatocellular carcinoma and can be served as an independent prognostic factor. J Hepatol 41: 104-111, 2004.

23. Peck-Radosavljevic M: Back to basics: Staging and prognosis in HCC for medical oncologist. J Hepatol 56: 488-489, 2012.

24. Chen JS, Wang Q, Fu XH, et al: Involvement of $\mathrm{PI} 3 \mathrm{~K} / \mathrm{PTEN} / \mathrm{AKT} / \mathrm{mTOR}$ pathway in invasion and metastasis in hepatocellular carcinoma: association with MMP-9. Hepatol Res 39: 177-186, 2009. 
25. Chen R, Cui J, Xu C, et al: The significance of MMP-9 over MMP-2 in HCC invasiveness and recurrence of hepatocellular carcinoma after curative resection. Ann Surg Oncol 19 (Suppl 3): S375-S384, 2012.

26. Yang J and Weinberg RA: Epithelial-mesenchymal transition: at the crossroads of development and tumor metastasis. Dev Cell 14: 818-829, 2008.

27. van Zijl F, Zulehner G, Petz M, et al: Epithelial-mesenchymal transition in hepatocellular carcinoma. Future Oncol 5: $1169-1179,2009$.

28. Lee TK, Poon RT, Yuen AP, et al: Twist overexpression correlates with hepatocellular carcinoma metastasis through induction of epithelial-mesenchymal transition. Clin Cancer Res 12: 5369-5376, 2006.
29. Lee S, Lee HJ, Kim JH, Lee HS, Jang JJ and Kang GH: Aberrant $\mathrm{CpG}$ island hypermethylation along multistep hepatocarcinogenesis. Am J Pathol 163: 1371-1378, 2003

30. Liu J, Lian Z, Han S, et al: Downregulation of E-cadherin by hepatitis B virus $\mathrm{X}$ antigen in hepatocellullar carcinoma. Oncogene 25: 1008-1017, 2006.

31. Yang MH, Chen CL, Chau GY, et al: Comprehensive analysis of the independent effect of twist and snail in promoting metastasis of hepatocellular carcinoma. Hepatology 50: 1464-1474, 2009

32. Zuo JH, Zhu W, Li MY, et al: Activation of EGFR promotes squamous carcinoma SCC10A cell migration and invasion via inducing EMT-like phenotype change and MMP-9-mediated degradation of E-cadherin. J Cell Biochem 112: 2508-2517, 2011. 